\title{
Value of susceptibility-weighted MR imaging (SWI) in the detection of developmental venous anomaly
}

\author{
Mohamed Saied Abdelgawad*io and Rasha Abdelhafiz Aly
}

\begin{abstract}
Background: Developmental venous anomaly (DVA) is probably the most common anomaly of the intracranial vasculature. DVAs consist of multiple, radially oriented dilated medullary veins that converge into a transcerebral vein. Susceptibility-weighted imaging (SWI) is a high spatial resolution 3D gradient-echo MRI sequence with phase post-processing that accentuates the paramagnetic properties of blood products such as deoxyhemoglobin, intracellular methemoglobin, and hemosiderin. Its high sensitivity to hemorrhagic particles by means of susceptibility dephasing effects within the veins allow for the accurate detection, grading, and monitoring of brain venous anomalies. In this review, we evaluated the prevalence of the brain DVAs identified by SWI in many patients who had undergone magnetic resonance imaging (MRI) with contrast administration. All images were independently reviewed by two radiologists who were blinded to other MR imaging finding. It is hoped that as SWI becomes more widely available, it will provide additional diagnostic and prognostic information that will improve the care and outcome of patients with DVAs.
\end{abstract}

Results: A total of 29 DVAs were observed with its prevalence 2.8\%. The DVA caputs had mostly deep localization in about $44.8 \%$ of our DVA cases. SWI proved excellent demonstration of DVAs with the sensitivity, specificity, positive predictive value (PPV), and negative predictive value (NPV) were significantly higher than the other non-contrast MR sequences. On SWI, DVA is delineated as a signal void lesion with the normal cerebral veins.

Conclusion: Susceptibility-weighted imaging (SWI) is valuable in the diagnosis of DVA and should be included in routine MR assessment of the brain.

Keywords: Developmental venous anomaly (DVA), Magnetic resonance imaging (MRI), Susceptibility-weighted imaging (SWI)

\section{Background}

Developmental venous anomaly (DVA), also referred to as cerebral medullary venous malformation, is the most frequently encountered cerebral vascular malformation. DVA consists of radially oriented, dilated veins (caput medusae appearance) that converge into an enlarged transcortical or subependymal draining vein [1]. DVA is typically diagnosed incidentally during imaging investigations as contrastenhanced MR imaging for unrelated symptoms [2].

* Correspondence: mselgawad@yahoo.com

Radiology Department, National Liver Institute, Menoufia University, Shebin Elkoum, Egypt

\section{Springer Open}

Although the parenchyma drained by a DVA is usually normal brain tissue, parenchymal abnormalities have been reported in the vicinity of a DVA. Possibly thrombosis or stenosis of the draining vein of DVA can cause a hemorrhagic event [3]. DVA is a low-flow vascular malformation that is not well imaged with conventional MR sequences. Small DVA can be easily missed and therefore contrast-enhanced magnetic resonance imaging (MRI) better allows the detection of DVA [4].

The contrast-enhanced (CE) study increases scan time and cost. Also, it may result in complications such as nephrogenic systemic fibrosis; therefore, it is 
contraindicated in patients with severe renal dysfunction [5]. Subsequently, non-contrast enhanced MR technique is required for cerebral venous malformation detection and lesion characterization [6].

Susceptibility-weighted imaging (SWI) is a novel high spatial resolution three-dimensional (3D) fully velocity MR technique compensated gradient-echo (GE) MRI sequence with an additional phase post-processing that accentuates the paramagnetic properties of blood products $[7,8]$. SWI has been found to obtain clinically useful complementary information to conventional spinecho MRI sequences [9].

By using paramagnetic deoxy-hemoglobin (deoxy-Hb) in the cerebral veins as an intrinsic contrast agent, SWI can demonstrate the normal cerebral veins and cerebral venous abnormality without contrast administration [10]. So, it was originally called "High-Resolution Blood oxygen level dependent (BOLD) Venography" providing an increased visibility of the venous vasculature in the brain [11].

The goal of our study is to assess the diagnostic value of SWI in comparison with T2-weighted, FLAIR, and contrast-enhanced images for DVA detection.

\section{Methods}

\section{Data collection}

In this study, after obtaining the approval of local ethics committee, we reviewed reports from a consecutive series of brain MRIs with intravenous contrast administration searching for brain DVAs performed over a 1year period at our institution. A few patients underwent MRI as a routine checkup. However, in most cases, the MRI was done for various clinical reasons such as headache, seizures, tinnitus, and dizziness. We evaluated the clinical symptoms and MR findings of DVAs.

\section{MR imaging data acquisition}

All MR examinations were performed at a 1.5 Tesla MR scanner (Siemens, Magnetom Avanto, 32 channels). MR protocol included T2-weighted imaging, fluid-attenuated inversion recovery (FLAIR), T1-weighted imaging, SWI, and CE T1-weighted imaging respectively (Table 1 ). The contrast agent was Gadopentetate dimeglumine (GdDTPA).

SWI was acquired by using a fully velocitycompensated, three-dimensional, GRE sequence. All images were obtained in the same axial plane. Subsequently, 2-mm minIP images were obtained. The sequence, along with entire image processing, was automated on Siemens MR scanner platforms. The SWI and minIP images were uploaded and made available on the picture archiving and communication (PACS) system.

In SWI, the magnitude and phase MR data are approached, and a phase mask is produced. Multiplying these with the original magnitude images produce a final magnitude SWI dataset. Both magnitude and phase information are needed for proper tissue characterization. Consequently, the continuity of tortuous veins across the slices could be identified.

SWI sequences have some intrinsic disadvantages. Unfavorable magnetic susceptibility sources that produce artifacts occurring at air-tissue interfaces such as the sinuses limit imaging of the nearby regions. Also, the blooming artifact may sometimes lead to extreme tissue signal cancellation and loss of anatomical borders.

All MRI images were read independently by two radiologists, including at least one senior-level radiologist. In cases of discrepancy, two reviewers reached a consensus later.

\section{Diagnostic criteria using MRI}

The diagnostic criteria of DVA on contrast-enhanced MRI and SWI were considered if the following criteria met presence of radially oriented, dilated veins that open into an enlarged transcortical or a subependymal collecting vein. The cluster of veins in DVA had "spoke-wheel" or "caput medusae" configuration. Assessments also included the presence of hyperintensity adjacent to DVA on FLAIR image in few cases as a potential sign of venous congestion and hypertension.

\section{Exclusion criteria}

We exclude cases with associated vascular malformations as cavernous angioma (CA) to assess the sensitivity of SWI in the detection of non-complicated DVA through cancelling the effect of potential signal-intensity

Table 1 MR imaging parameters

\begin{tabular}{|c|c|c|c|c|c|c|c|}
\hline & TR (ms) & TE (ms) & Section thickness (mm) & Intersection gap (mm) & FOV $(\mathrm{cm})$ & Matrix & Imaging time \\
\hline T1 & 550 & 15 & 3 & 0.3 & 18 & $256 \times 256$ & $3 \min 23 \mathrm{~s}$ \\
\hline $\mathrm{T} 2$ & 3000 & 120 & 3 & 0.3 & 18 & $256 \times 256$ & $2 \min 51 \mathrm{~s}$ \\
\hline FLAIR & 80000 & 138 & 3 & 0.3 & 36 & $288 \times 288$ & $3 \min 15 s$ \\
\hline SWI & 6400 & 30 & 2.4 & 0.2 & 28 & $224 \times 224$ & $3 \min 35 s$ \\
\hline Contrast T1 & 550 & 15 & 3 & 0.3 & 18 & $256 \times 256$ & $3 \min 23 \mathrm{~s}$ \\
\hline
\end{tabular}

T1WI T1-weighted image, T2WI T2-weighted image, FLAIR fluid-attenuated inversion recovery, SWI susceptibility-weighted image, TR repetition time, TE echo time, FOV field of view 
changes related to adjacent non-related parenchymal pathology.

\section{Statistical analysis}

The data collected were tabulated and analyzed by the Statistical Package for Social Science (SPSS) version 26.0. The data were presented as the mean \pm standard deviation, median (minimum-maximum), and percentage. The sensitivity, specificity, positive predictive value (PPV), and negative predictive value (NPV) of SWI sequence for DVA detection were determined.

\section{Results}

A total of 1038 adult individuals underwent MRI study with contrast administration as well as SWI sequence over a 1-year period; 29 DVAs were detected. The prevalence of DVAs has been determined to be $2.8 \%$ of the total examined cases. All cases were presented by clinical findings suggestive of brain lesions such as headache, seizures, or dizziness. Our cases in the study with DVA include 21 males and eight females with their age ranging from 22 to 48 years old (mean about 38.2 years).

According to the DVA size, 29 DVA cases are divided into three groups: small-, medium-, and large-sized DVA representing about 18 cases $(62.1 \%)$, ten cases $(34.5 \%)$, and one case (3.4\%) respectively (Fig. 1) based on the visibility of DVA collecting vein on both contrast-enhanced MR and SWI with average diameter around $1 \mathrm{~mm}, 2 \mathrm{~mm}$, and $3 \mathrm{~mm}$ for small, medium, and large DVA respectively. Although the parenchyma drained by DVAs is generally considered to be normal within our cases, associated hyperintensity on FLAIR sequence (non-hemorrhagic signal intensity alteration) within the drainage territory of a DVA was identified in three of 29 cases (10.3\%). No diffusion restriction was observed on DWI/ADC image in the area of signalintensity abnormality excluding cerebral infarction. It is hypothesized that mild chronic local venous congestion and hypertension leads to this phenomenon.

No evidence of accompanying brain pathologies such as intracerebral hemorrhage, cerebral infarction, or leukomalacia. Also, no calcification deposit was noted in the surrounding brain parenchyma or the walls of the DVA.

In our study, the non-contrast brain MRI as T1- or T2-weighted images did not provide a definitive diagnosis of DVA. T2-weighted images showed false negative in $27 / 29$ proved DVA cases (93.1\%). Considering FLAIR images, true positive result was noted in about $12 / 29$ (41.4\%) with false negative result in about $17 / 29$ cases (58.6\%).

Based on contrast-enhanced MRI conducted with 3D SPGR sequence, SWI showed definite diagnosis of DVA in our proved DVA cases involving small DVAs with $100 \%$ true positive result. So, SWI presented an overall sensitivity and specificity for DVA detection ranging from 96.6 to $100 \%$ with significantly high positive predictive value (PPV) and negative predictive value (NPV) representing about $96.66 \%$ and $93.1 \%$ respectively in comparison to the other non-contrast MR sequences (Fig. 2) raising its global diagnostic accuracy. DVA is delineated on SWI as a signal void lesion with the normal cerebral veins.

Among the 29 DVA cases, 25 (86.2\%) DVAs were in either lobes of the supratentorial brain and four (13.8\%) were in the infratentorial region. We observed that 28 of 29 cases $(96.6 \%)$ with a single DVA and only one case (3.4\%) of double DVAs (supra- and infratentorial) (Fig. 4).

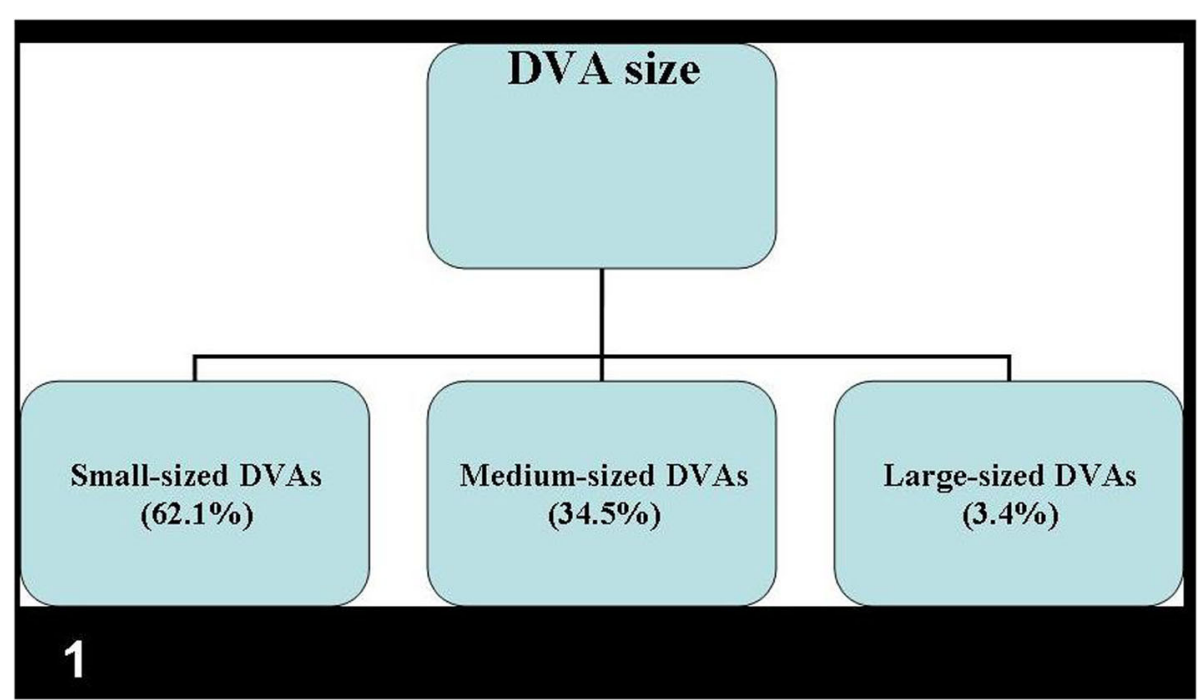

Fig. 1 Prevalence of developmental venous anomalies by size 


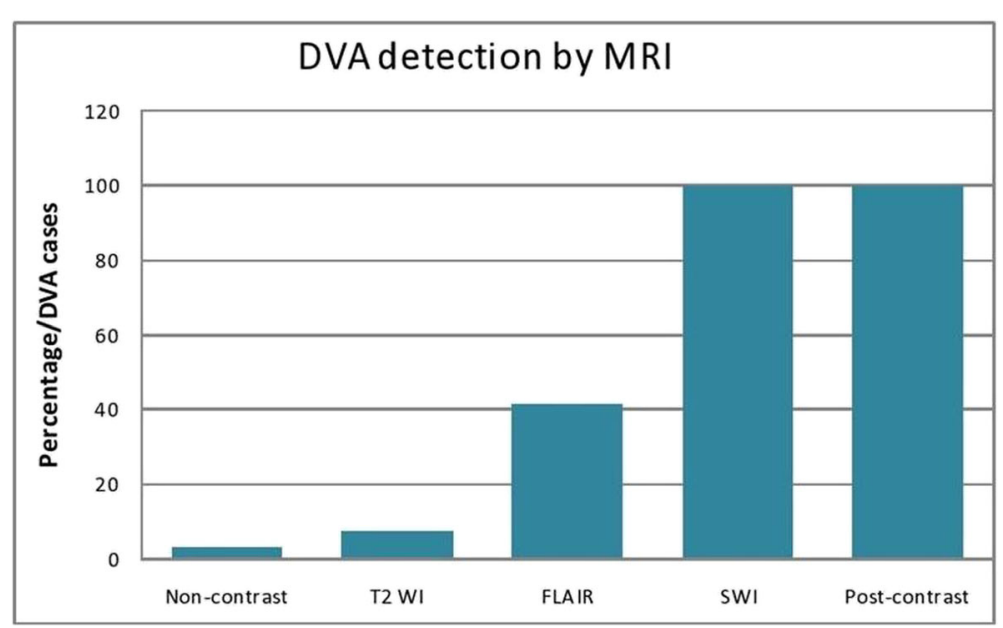

Fig. 2 Detection of DVA by different MRI sequences in relation to the T1W contrast enhanced sequence

About $89.7 \%$ of DVA cases had only one draining vein to the superficial or deep venous system according to the DVA location.

Of the supratentorial DVAs, 19 of 25 cases $(76 \%)$ had DVA located in the left cerebral hemisphere (Figs. 3, 4, 5, 6, and 7), while six cases (24\%) in the right cerebral hemisphere (Fig. 6). The supratentorial DVAs were frequently located in the frontal lobe, representing about 14 of 25 cases (48.3\% of total DVA cases). Of the infratentorial DVAs, all four cases (13.8\% of total DVA cases) were in the cerebellum with no detectable brain stem lesion. The previously mentioned infratentorial DVAs are seen draining centrally to the nearby deep venous system within the quadrigeminal cistern (including the internal cerebral vein, basal vein of Rosenthal, or the great cerebral vein of Galen). In Table 2, we have shown intracranial localizations and frequencies of DVAs within our study.

The brain parenchymal areas where DVA caputs were spread and drained, including the following areas: deep, subcortical, or juxtacortical. The subcortical area has been defined as the area below the juxtacortical region that is not adjacent to the ventricular wall. The deep has been defined as the area adjacent to the ventricular system. The most DVA caputs were deep representing about $44.8 \%$ of our total DVA cases (Table 3 ).

\section{Discussion}

The cerebral vascular malformations are mostly classified into four categories: Developmental venous anomaly (DVA), arteriovenous malformation (AVM), capillary telangiectasia, and cavernous angioma (CA) $[12,13]$. Of these, DVA is the most common one, constituting about $60 \%$ of all vascular malformations, especially with the progresses of MR imaging and is mostly incidental on neuroimaging [14]. Hammoud et al. [15] thought that
DVAs are benign entities and likely to become asymptomatic, although headache and epilepsy were reported.

The exact prevalence of DVA is nearly unidentified, but autopsy studies had reported an incidence of 2-3\% in the general population [16]. In our study, DVAs were demonstrated a $2.8 \%$ incidence, which is also like those observed by Cho et al. [1], who reported a $2.6 \%$ incidence in 1068 brain MR examinations.

DVAs are characterized by a cluster of venous radicles that converge into a draining vein, resulting in the typical caput medusae or umbrella-like appearance. To join either the superficial or deep venous system, the collecting vein crosses a variable length of cerebral or cerebellar parenchyma [17].

Several studies thought that the incidence of two or more DVAs existing in different regions of the brain simultaneously about $1.2-16.3 \%$ of cases [18]. Most our studied cases were noted with a single DVA with the prevalence of double DVAs was only $3.4 \%$, which is in accordance with Lee et al. [19], who found that $1.6 \%$ of their cases had three DVAs. Faure et al. [3] also reported that most DVAs were solitary.

Of the total 29 DVA cases, DVAs are mostly located in the supratentorium (86.2\%), with a clear frontal predominance (48.3\%). The parietal lobe showed the second most frequent location representing about $31 \%$. While the temporal lobe showed only $6.9 \%$ with no occipital or basal ganglia DVAs were noted. Regarding the infratenotrial region, DVAs were accounted about $13.8 \%$ with only cerebellar location. Various articles exhibited similar findings to our study. The study by Garner et al. [20], of 100 cases with DVA diagnosed by DSA, CT, and MRI examinations, showed that DVAs are predominant in the frontal location (42\%), while the parietal and cerebellar locations representing about $24 \%$ and $14 \%$ respectively. To Huang et al. [21], DVAs were frequently been 

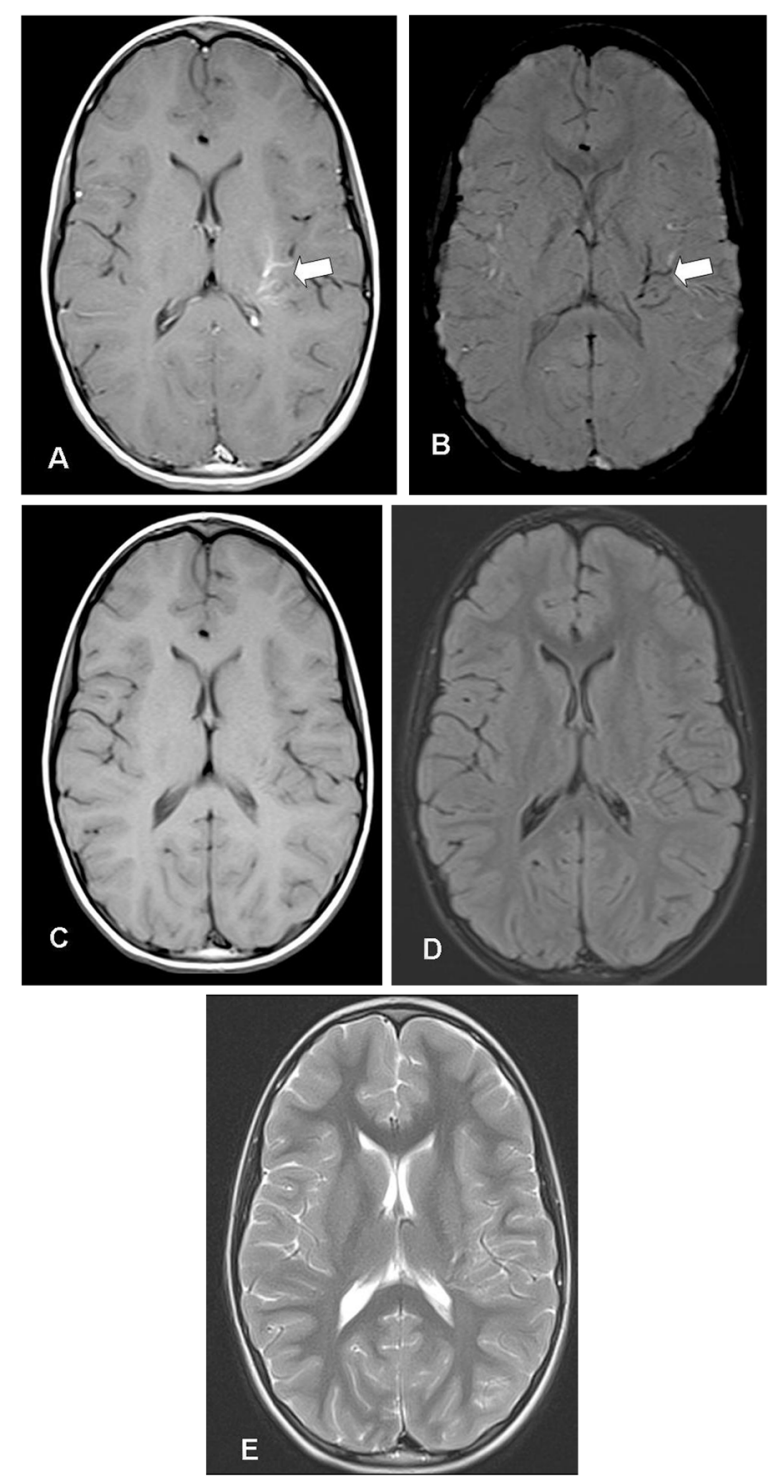

Fig. 3 Contrast-enhanced T1W (a) and SWI (b) show a DVA in the left temporal lobe (arrow). On non-contrast T1W (c), FLAIR (d), and $\mathrm{T} 2 \mathrm{~W}(\mathbf{e})$, there is no definite abnormality at the left temporal lobe

found in the frontal lobe (40\%) and the cerebellum (27\%) and were less often in the parietal lobe (15\%). Wilms et al. [22] reported that the prevalence of DVA locations to be as follows: the frontal (38\%), the cerebellar $(38 \%)$, the parietal $(10 \%)$, and the occipital regions (10\%).

DVAs can be located subcortically, juxtacortically, and deeply (or paraventricularly) with the corresponding cases for each area; 9/29 (31.1\%), 7/29 (41.1\%), and $13 / 29(44.8 \%)$ respectively. This categorization was first suggested by Valavanis et al. [23] and Ostertun et al.'s [24] studies. Both showed that DVA caputs were localized predominantly in the subcortical
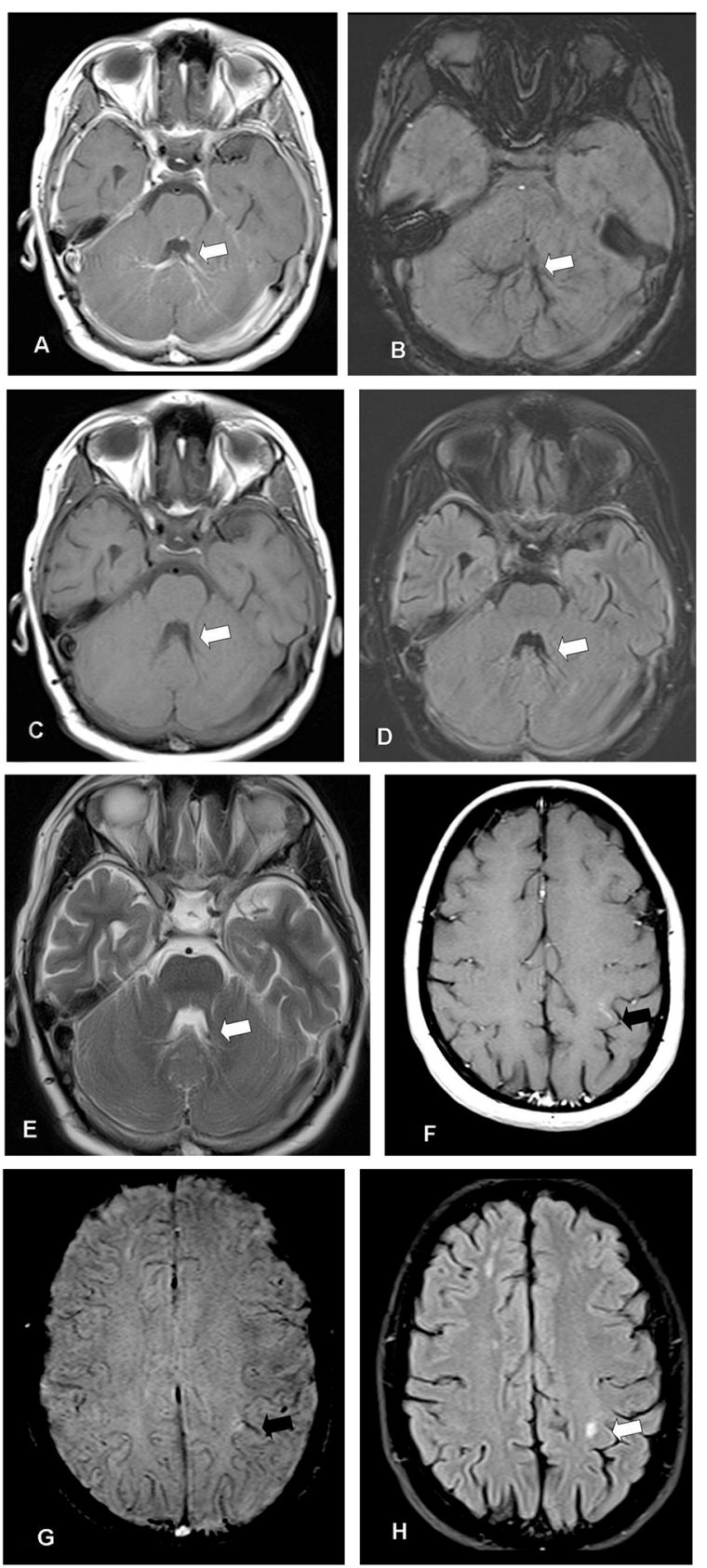

Fig. 4 Contrast-enhanced T1W (a) and SWI (b) show large cerebellar DVA (white arrow). On non-contrast T1W (c), FLAIR (d), and T2W (e), the lesion is less clearly visible (white arrow). The same patient shows coexistent supra-tentorial left posterior parietal smaller DVA (black arrow) seen on post-contrast $\mathrm{T1}(\mathbf{f})$ and SWI (g) with associated parenchymal hyperintense signal on FLAIR (h). Other nonspecific white matter small ischemic foci seen at the right frontal lobe with no associated gross vascular anomalies (h)

region representing $57.1 \%$ and $61.9 \%$ respectively. However, our findings were matched with the study performed by Gökçe et al. [13], who observed predominant deep parenchymal DVA location, 54.5\%, followed by the subcortical area, $17 \%$. 


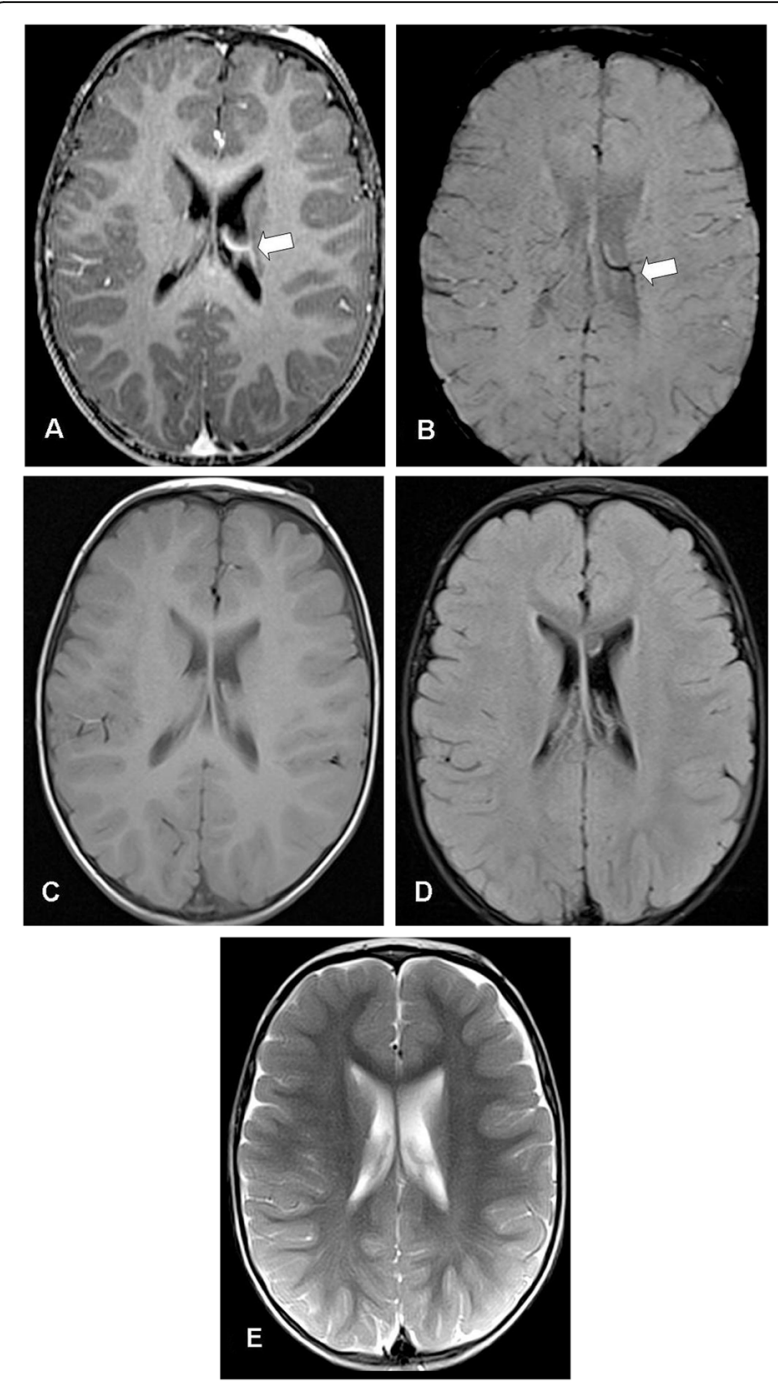

Fig. 5 Contrast-enhanced T1W (a) and SWI (b) show a small DVA in the left frontal lobe (arrow). The lesion is less clearly demonstrated on non-contrast T1W (c), FLAIR (d), and T2W (e) with no definite abnormality at the left frontal lobe

Various parenchymal abnormalities and lesions can accompany DVAs [13]. The parenchymal abnormalities within our study are relatively few, but it may be related to selection bias and sample size. In our study, three of 29 DVA cases (10.3\%) displayed hyperintensity on FLAIR sequence within the drainage territory of a DVA with no identified diffusion restriction. Few previous studies have estimated this association. In a study performed by Ruiz et al. [25], signal-intensity abnormalities in the drainage territory was $28.3 \%$ of 60 DVAs evaluated by MRI, more than twice the frequency reported in our study. However, the etiology of signal-intensity abnormalities related to DVAs is relatively unclear; Santucci et al. [4] reported that the abnormal FLAIR high signal intensity may represent edema or gliosis caused
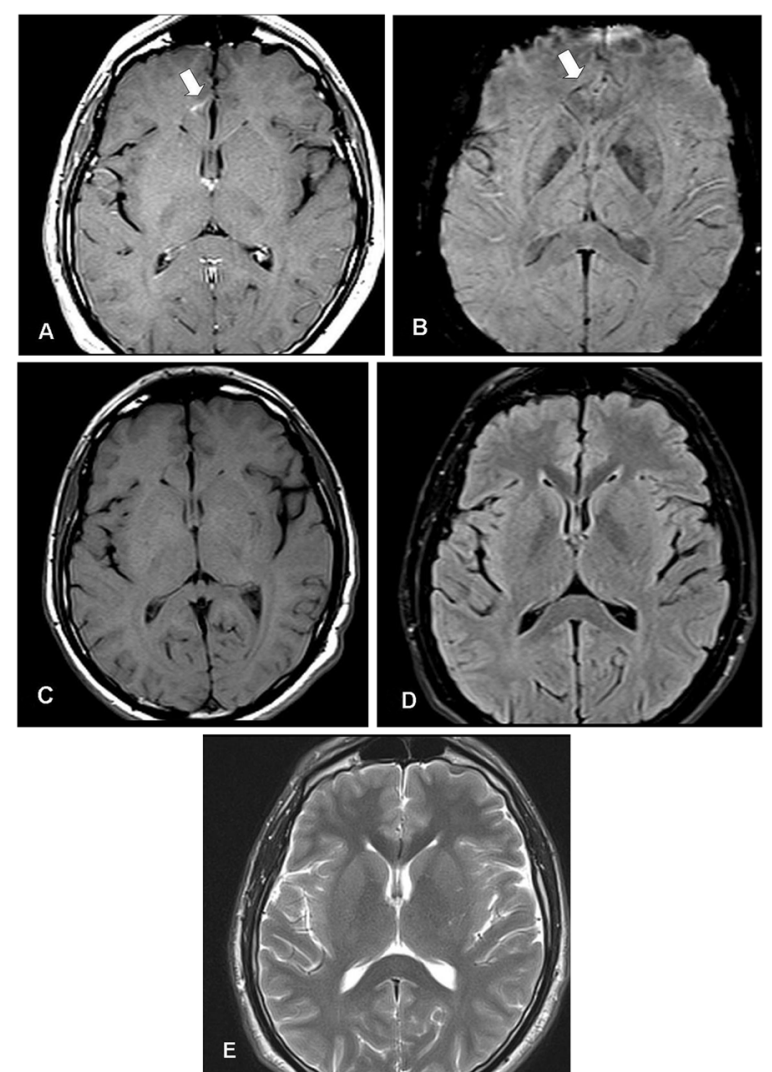

Fig. 6 Contrast-enhanced T1W (a) and SWI (b) show a small DVA in the right frontal lobe (arrow). The lesion is less clearly demonstrated on non-contrast T1W (c), FLAIR (d), and T2W (e) with no definite abnormality at the right frontal lobe

by chronic mild venous congestion and hypertension as a result of the anomalous venous drainage.

Our study showed no evidence of accompanying brain pathologies such as intracerebral hemorrhage, cerebral infarction, or leukomalacia. Also, no calcification deposit was noted in the surrounding brain parenchyma or the walls of the DVA.

To $\mathrm{Ku}$ et al. [26], intracerebral hemorrhage is a rare DVA complication and predominately described in patients with coexisting vascular malformations. McLaughlin et al. [27], in their observational study of 80 patients with symptomatic DVAs, found an annual hemorrhage rate of $0.68 \%$ per year retrospectively and $0.34 \%$ per year in the prospective study. Garner et al. [20] revealed that the hemorrhages that associated with DVA are usually small and do not lead to high mortality. The previous studies are matched with our results regarding unidentified cerebral hemorrhage within our DVA cases.

DVAs were found to be rare before the developments in MR imaging, as had to be undetectable on unenhanced MRI as well as on MRA image, unless they were associated with a CM. Nowadays, they are seen with 


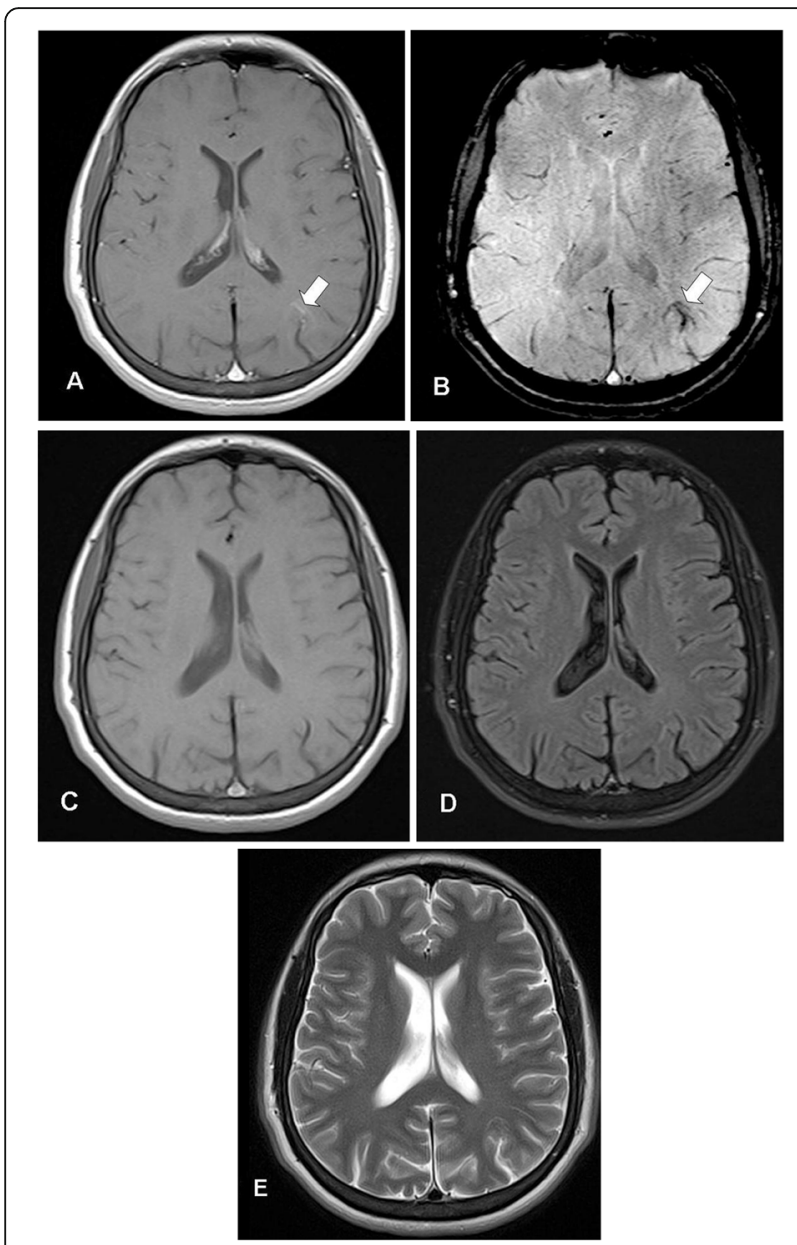

Fig. 7 Contrast-enhanced T1W (a) and SWI (b) show a small DVA in the left parietal lobe (arrow). The lesion is less clearly demonstrated on non-contrast T1W (c), FLAIR (d), and T2W (e) with no definite abnormality at the left parietal lobe

Table 2 Intracranial localizations and numerical distributions of DVAs

\begin{tabular}{|c|c|c|}
\hline DVA localizations & No. of cases & $\% /$ DVA cases \\
\hline \multicolumn{3}{|c|}{ Supratentorial region (25/29) } \\
\hline o Frontal Lobe & $14(56 \%)$ & $48.3 \%$ \\
\hline - Parietal Lobe & $9(36 \%)$ & $31 \%$ \\
\hline - Temporal Lobe & $2(8 \%)$ & $6.9 \%$ \\
\hline o Occipital Lobe & 0 & $0.0 \%$ \\
\hline - Basal Ganglia & 0 & $0.0 \%$ \\
\hline \multicolumn{3}{|c|}{ Infratentorial region (4/29) } \\
\hline - Cerebellum & $4(100 \%)$ & $13.8 \%$ \\
\hline o Brain stem & 0 & $0.0 \%$ \\
\hline Intraventricular location & 0 & $0.0 \%$ \\
\hline Total & 29 & 100.0 \\
\hline
\end{tabular}

DVA developmental venous anomaly
Table 3 Localizations and frequencies of DVA caputs

\begin{tabular}{lll}
\hline Localizations of DVA caputs & No. of cases & $\% / D V A$ cases \\
\hline Deep & 13 & $44.8 \%$ \\
Subcortical & 9 & $31.1 \%$ \\
Juxtacortical & 7 & $24.1 \%$ \\
Total & 29 & 100.0
\end{tabular}

DVA developmental venous anomaly

high frequency as incidental finding at contrastenhanced MRI [28].

Susceptibility-weighted imaging (SWI), an increasingly used MR sequence in recent years with a high sensitivity effect, can better detect vascular anomalies such as the DVA and has been shown to be more sensitive than conventional $\mathrm{T} 2 *$ sequence in the detection of vascular structures [10]. SWI can demonstrate the whole vascular structure well and typically have a low signal intensity due to the blood oxygen level-dependent (BOLD) effect in the dilated medullary and draining veins [4].

We consider that SWI has a high potential for the detection of non-complicated DVAs including the small lesions without contrast-enhanced MR study. SWI in our study showed $100 \%$ true positive result with consequent high global diagnostic accuracy. So, invasive imaging as conventional cerebral angiography as well as contrastenhanced MR imaging are not required for their confirmation and mostly reserved for complicatd cases or to exclude associated vascular lesions.

\section{Limitations}

Although SWI has been shown to be superior to conventional MRI in vessel imaging and detection of hemorrhagic lesions, some limitations have still been found. The long acquisition times required to obtain an SWI image, which makes movement artifacts likely because of patient discomfort, with subsequently distortion of SWI findings. SWI is prone to air-tissue artifacts with resultant susceptibility artifacts, such as bone structures which can distort image findings. In addition, limitation of the present study is that there may have been selection biases. SWI is not limited to lesion detection and characterization.

\section{Conclusion}

Developmental venous anomaly (DVA) is likely the most common anomaly of the intracranial vasculature and represents an anatomical variant of venous drainage rather than a true malformation. DVAs can be observed in both the supra- and infratentorial brain parenchyma. They have a relatively benign nature with a low incidence of hemorrhage. SWI, which is a combination of GE techniques with phase information, represents a useful tool for the detection and characterization of vascular 
malformations with no need to contrast administration. The uncomplicated DVAs may not be detected by conventional MR sequences as most patients presented with nonspecific symptoms. So, SWI should be included as a routine MR sequence in diagnosing different cerebral vascular pathologies to avoid missing lesions that may lead to future complications.

\section{Abbreviations \\ DVA: Developmental venous anomaly; SWI: Susceptibility-weighted imaging; 3D: Three dimensional; MRI: Magnetic resonance imaging; CE: Contrast- enhanced; deoxy-Hb: Deoxy-hemoglobin; BOLD: Blood oxygen level dependent; FLAIR: Fluid-attenuated inversion recovery; Gd- DTPA: Gadopentetate dimeglumine; minIP: Minimum intensity projection; PACS: Picture archiving and communication system; T1Wl: T1-weighted image; T2Wl: T2-weighted image; TR: Repetition time; TE: Echo time; FOV: Field of view; SPSS: Statistical Package for Social Science; PPV: Positive predictive value; NPV: Negative predictive value; CM: Cavernous malformation; CA: Cavernous angioma}

\section{Acknowledgements}

Not applicable.

\section{Authors' contributions}

MSA carried out cases on workstation and selection of research cases, prepared the figures for cases demonstration, and wrote and reviewed the research. RAA wrote the research, shared in selection of the cases, and shared in figures preparation of cases. All authors read and approved the final manuscript.

\section{Funding}

This study had no funding from any resource.

\section{Availability of data and materials}

The datasets used and/or analyzed during the current study are available from the corresponding author on reasonable request.

\section{Ethics approval and consent to participate}

All procedures followed were in accordance with the ethical standards of the responsible committee on human experimentation (Institutional Review Board (IRB)" of National Liver Institute Menoufia University and with the Helsinki Declaration of 1964 and later versions. Committee's reference number is unavailable (NOT applicable). No consent was obtained from the patients since it was a retrospective study.

\section{Consent for publication}

All patients included in this research gave written informed consent to publish the data contained within this study.

\section{Competing interests}

The authors declare that they have no competing interests.

Received: 13 April 2020 Accepted: 27 May 2020

Published online: 09 June 2020

\section{References}

1. Cho S, Choi D, Ryu H, Shin H, Kim J, Choi H, Park M, Son S (2014) Susceptibility-weighted mr imaging for the detection of developmental venous anomaly: comparison with T2 and FLAIR imaging. JKSMRM 18(3): 200-207

2. Takasugi M, Fujii S, Shinohara Y, Kaminou T, Watanabe T, Ogawa T (2013) Parenchymal hypointense foci associated with developmental venous anomalies: evaluation by phase-sensitive MR imaging at 3T. AJNR Am J Neuroradiol 34:1940-1944

3. Faure M, Voormolen M, Van der Zijden T, Parizel P (2014) Developmental venous anomaly: MR and angiographic features. JBR-BTR 97:17-20

4. Santucci G, Leach J, Ying J, Leach S, Tomsick T (2008) Brain parenchymal signal abnormalities associated with developmental venous anomalies: detailed MR imaging assessment. AJNR Am J Neuroradiol 29:1317-1323
5. Daftari Besheli L, Aran S, Shaqdan K, Kay J, Abujudeh H (2014) Current status of nephrogenic systemic fibrosis. Clin Radiol 69:661-668

6. Halefoglu A, Yousem D (2018) Susceptibility weighted imaging: clinical applications and future directions. World J Radiol 10(4):30-45

7. Tong K, Ashwal S, Obenaus A, Nickerson J, Kido D, Haacke E (2008) Susceptibility-weighted MR imaging: a review of clinical applications in children. AJNR Am J Neuroradiol 29:9-17

8. Bosemani T, Poretti A, Huisman T (2014) Susceptibility-weighted imaging in pediatric neuroimaging. J Magn Reson Imaging 40:530-544

9. Haacke E, Mittal S, Wu Z, Neelavalli J, Cheng Y (2009) Susceptibilityweighted imaging: technical aspects and clinical applications. Part 1. Am J Neuroradiol 30(1):19

10. Tsui Y, Tsai F, Hasso A, Greensite F, Nguyen B (2009) Susceptibility-weighted imaging for differential diagnosis of cerebral vascular pathology: a pictorial review. J Neurol Sci 287:7-16

11. Chavhan G, Babyn P, Thomas B, Shroff M, Haacke E (2009) Principles, techniques, and applications of T2*-based MR imaging and its special applications. Radiographics 29(5):1433

12. San Millan Ruiz D, Gailloud P (2010) Cerebral developmental venous anomalies. Childs Nerv Syst 26:1395-1406

13. Gökçe E, Acu B, Beyhan M, Çelikyay F, Çelikyay R (2014) Magnetic resonance imaging findings of developmental venous anomalies. Clin Neuroradiol 24: 135-143

14. Pereira V, Geibprasert S, Krings T (2008) Pathomechanisms of symptomatic developmental venous anomalies. Stroke 39:3201-3215

15. Hammoud D, Beauchamp N, Wityk R, Yousem D (2002) Ischemic complication of a cerebral developmental venous anomaly: case report and review of the literature. J Comput Assist Tomogr 26:633-636

16. Mullaguri N, Battineni A, Krishnaiah B (2019) Developmental venous anomaly presenting with spontaneous intracerebral hemorrhage, acute ischemic stroke, and seizure. Cureus 11(8):e5412

17. Ruiz D, Yilmaz H, Gailloud P (2009) Cerebral developmental venous anomalies: current concepts. Ann Neurol 66:271-283

18. Lee M, Kim MS (2012) Image findings in brain developmental venous anomalies. J Cerebrovasc Endovasc Neurosurg 14:37-43

19. Lee C, Pennington M, Kenney C (1996) MR evaluation of developmental venous anomalies: medullary venous anatomy of venous angiomas. AJNR Am J Neuroradiol 17:61-70

20. Garner T, Curling O, Kelly D, Laster D (1991) The natural history of intracranial venous angiomas. J Neurosurg 75:715-722

21. Huang Y, Patel S, Robbins A, Chaudhary M (1984) Cerebral venous malformation and a new classification of cerebral vascular malformations. In Kapp JP, Schmidek HH (eds) The cerebral venous system and its disorders. Grune \& Stratton, Orlando, pp 373-474

22. Wilms G, Demaerel P, Marchal G, Baert AL, Plets C (1991) Gadolinium enhanced MR imaging of cerebral venous angiomas with emphasis on their drainage. J Comput Assist Tomogr 15:199-206

23. Valavanis A, Wellauer J, Yasargil M (1983) The radiological diagnosis of cerebral venous angioma: cerebral angiography and computed tomography. Neuroradiology 24:193-199

24. Ostertun B, Solymosi L (1993) Magnetic resonance angiography of cerebral developmental venous anomalies: its role in differential diagnosis. Neuroradiology 35:97-104

25. Ruiz DS, Delavelle J, Yilmaz H (2007) Parenchymal abnormalities associated with DVAs. Neuroradiology 49:987-995

26. Ku M, Rhee D, Park H, Kim D (2009) Repeated intracerebral hemorrhage from developmental venous anomaly alone. J Korean Neurosurg Soc 45(1): 46-49

27. McLaughlin M, Kondziolka D, Flickinger J, Lunsford S, Lunsford L (1998) The prospective natural history of cerebral venous malformations. Neurosurgery 43:195-200

28. Fok K, Holmin S, Alvarez H, Ozanne A, Krings T, Lasjaunias P (2006) Spontanous intracerebral hemorrhage caused by an unusual association of developmental venous anomaly and arteriovenous malformation. Interv Neuroradiol 12:113-121

\section{Publisher's Note}

Springer Nature remains neutral with regard to jurisdictional claims in published maps and institutional affiliations. 